\title{
Hemoptysis Due to Breath-Hold Diving Following Chemotherapy and Lung Irradiation
}

\author{
Markus Gutsche, MD and Ware G. Kuschner, MD
}

\begin{abstract}
Breath-hold diving, also known as free-diving, describes the practice of intentional immersion under water without an external supply of oxygen. Pulmonary hemorrhage with hemoptysis has been reported as a complication of immersion and breath-hold diving in young healthy athletes. We report the case of a 60 -year-old man with a history of radiation and chemotherapy for breast carcinoma, who developed the abrupt onset of hemoptysis in the setting of swimming and breath-hold diving. A computed tomography (CT) scan of the chest demonstrated an area of ground glass opacification, suggestive of pulmonary hemorrhage, superimposed on a background of reticular opacities within the prior radiation field. A follow-up CT scan of the chest, obtained 2 months after presentation, demonstrated resolution of the ground glass opacification, but persistence of fibrotic features attributable to prior radiation therapy. We postulate that prior irradiation of the chest resulted in lung injury and fibrosis which, in turn, rendered the affected region of the lung susceptible to "stress failure," due to an increase in the transcapillary pressure gradient arising from immersion and breath-hold diving. Patients with a history of lung injury resulting from chest irradiation should be cautioned about pulmonary hemorrhage and hemoptysis as a potential complication of swimming and breath-hold diving.
\end{abstract}

Keywords: Breath-hold diving; Diving injury; Hemoptysis; Immersion; Pulmonary capillary stress failure

Corresponding Author:

Ware G. Kuschner, MD

VA Palo Alto Heath Care System

380 I Miranda Avenue, Pulmonary Section,

Mail Code: IIIP

Palo Alto, CA 94304

Tel: (650) 493-5000, ext. 63544

Fax: (650) 852-3276

Email: kuschner@stanford.edu

Received: July 26, 2011

Revised: December 5, 2011

Accepted: December 7, $201 \mathrm{I}$

doi: $10.3121 / \mathrm{cmr} .2011 .1038$
A up blood. He had been in his usual state of health until the day of admission, when he went breath-hold diving in Monterey Bay, off the coast of northern California. At the time of his dives, the water temperatures usually average around $55^{\circ} \mathrm{F}$. The patient completed five dives, each to a depth of approximately 13 meters ( $\sim 40$ feet) and each lasting approximately 90 seconds. Hyperventilation preceded each dive, but no other maneuvers, such as glossopharyngeal insufflation ("lung packing"), were performed. The patient was unsure about diaphragmatic contractions performed against a closed glottis during immersion. After his fifth dive, he noted "slight irritation" in his chest that was immediately followed by a series of five coughs, over a period of 5 minutes. He expectorated an estimated $1 / 2$ to 1 tablespoon of blood with each cough; approximately, 4 tablespoons of blood in total. He noted wheezing but did not feel short of breath.

The patient's past medical history was significant for T2N3M0 stage IIIc invasive ductal carcinoma of the left breast, for which he had undergone a left modified radical mastectomy and axillary lymph node dissection 10 months prior to presentation. He had been treated with adjuvant chemotherapy 6 months prior to presentation that included doxorubicin, cyclophosphamide, and paclitaxel followed by adjuvant radiation therapy administered in 1.8 Gray (Gy) fractions, 


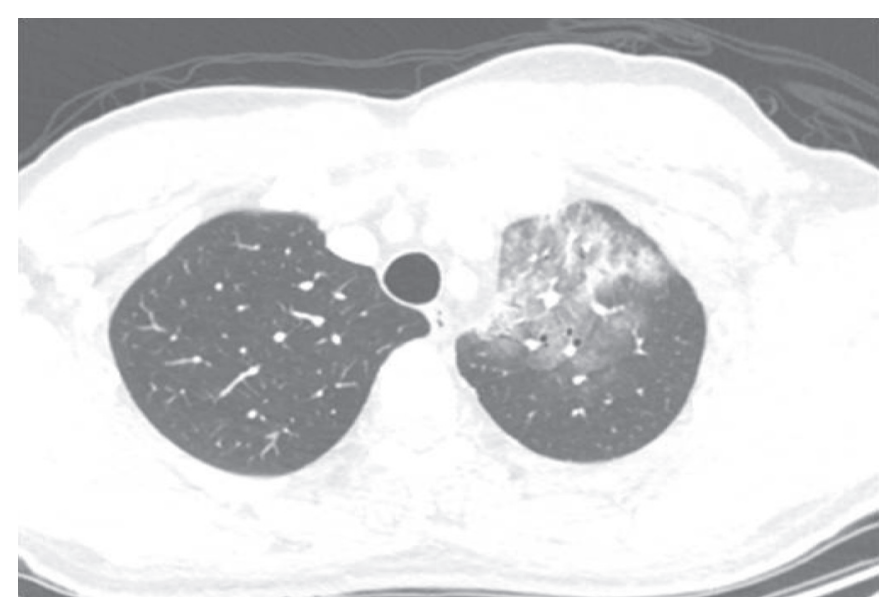

Figure 1: The chest CT angiogram obtained on presentation shows extensive ground glass opacification in the upper anterior left lung zone.

with 45 Gy to the left chest and 50.4 Gy to the supraclavicular region. The past medical history was otherwise unremarkable. $\mathrm{He}$ had a remote history of cigarette smoking. He was not taking any antithrombotic medication or non-steroidal antiinflammatory drugs. His spirometry prior to diagnosis with breast cancer was within normal limits. A limited transthoracic echocardiogram revealed a normal left ventricle and normal ejection fraction.

The patient presented to the emergency department approximately 4 hours after the event. He reported feeling well and appeared comfortable. The respiratory rate was 16 breaths per minute and heart rate was 47 beats per minute. The hemoglobin oxygen saturation was $100 \%$ while the patient was breathing ambient air. The physical examination was normal. A basic metabolic panel, complete blood count, erythrocyte sedimentation rate, and urinalysis were normal.

A chest radiograph demonstrated ill-defined patchy opacities in the left upper lobe that were not present on a chest radiograph obtained 6 months earlier. A computed tomography (CT) scan of the chest showed ground glass opacification and subpleural reticular opacities in the left upper lobe (figure 1). The chest CT angiogram did not demonstrate a pulmonary embolism.

The patient was admitted for observation. He experienced no further hemoptysis, remained asymptomatic, and on hospital day 3 he was discharged to home. The temporal relationship between the patient's breath-hold diving and the onset of hemoptysis, the absence of other causes of pulmonary hemorrhage, and the self-limited clinical course are consistent with pulmonary capillary stress failure secondary to immersion and breath-hold diving in the setting of recent chemotherapy and lung irradiation.

At follow-up 2 months later, the patient felt well and reported no further hemoptysis. A repeat CT scan of the chest demonstrated resolution of the ground glass opacification, but persistent subpleural reticular opacities and mild fibrotic changes were present (figure 2). The paired clinicalradiographic observations suggest that this patient experienced stress failure of the pulmonary capillary bed in a region of the lung previously injured by irradiation. The patient was advised to refrain from further breath-hold diving and immersion due to a presumptive increased risk of recurrent pulmonary hemorrhage.

\section{Discussion}

Breath-hold diving, also known as free diving (ie, diving under without an external oxygen supply), as well as swimming and immersion by itself can all result in lung injury, including pulmonary edema and alveolar hemorrhage. ${ }^{1}$ Several factors contribute to lung injury during breath-hold diving.

With progressively deeper descent underwater, ambient pressure rises, and gas in the lung is compressed resulting in decreased lung volume based on Boyle's law. When the total lung capacity (TLC) is reduced to residual volume (RV), intrathoracic pressure is equal to hydrostatic pressure, and lung volume cannot decrease further because of the rigidity of the thorax. ${ }^{2}$ As the chest wall approaches its elastic limit, a high negative transthoracic pressure develops, resulting in increased blood return to the thorax and an increase in pulmonary transcapillary wall pressure. Eventually, redistribution of blood to the chest may cause "stress failure" of the pulmonary capillaries, resulting in leakage of fluid and blood into the airspaces. ${ }^{3}$ In our case, given the relatively shallow diving depth of only 13 meters, pulmonary barotrauma of descent or "lung squeeze" seems less likely. However, other mechanisms during breath-hold diving and immersion or swimming may contribute to the pooling of blood in the

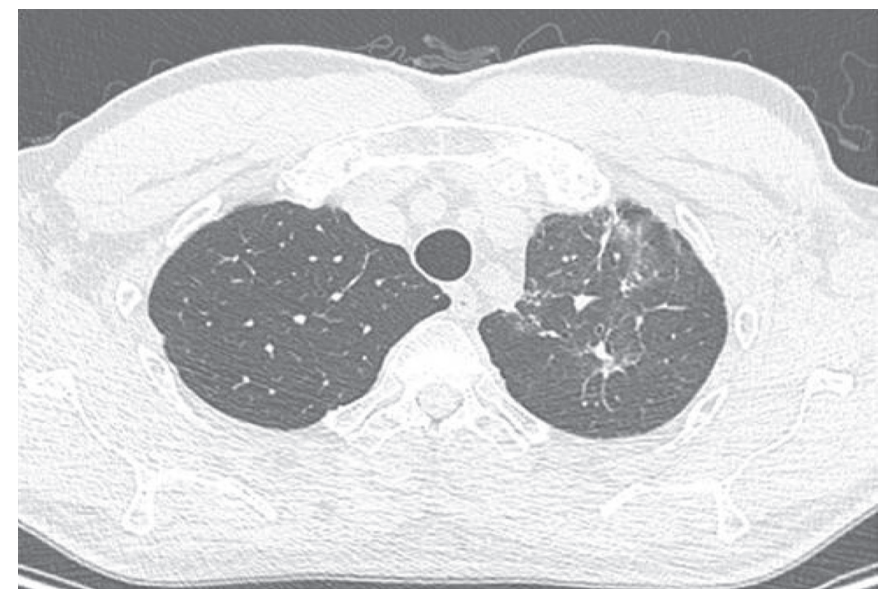

Figure 2: A repeat chest CT obtained 2 months after presentation shows mild irregularity of the left anterior pleural-parenchymal interface and mild interlobular septal thickening in the left lung apex which most likely represents post-radiation changes following left breast cancer. The ground glass opacification that was previously observed in the setting of acute pulmonary hemorrhage have resolved. 
lung, and in turn, capillary injury and leak. Exposure to ambient cool temperatures causes vasoconstriction with increases in preload and afterload contributing to pulmonary edema with hemoptysis. ${ }^{4}$ Exercise-induced pulmonary hypertension has been proposed as another possible factor contributing to capillary stress failure during exertion. ${ }^{5}$ Diaphragmatic movement against a closed glottis during diving causes a negative intra-alveolar pressure and can further exacerbate the bulging of pulmonary capillaries resulting in an increase in transcapillary pressure gradient that can lead to capillary rupture. ${ }^{6}$

We speculate that pre-existing focal lung damage from radiation therapy 3 months prior placed this patient at increased risk for capillary stress failure and alveolar hemorrhage. This is suggested by the finding of ground glass opacification present on the initial CT scan of the chest, consistent with alveolar hemorrhage, that was localized to a region of the lung that had been in the prior radiation field. The ground glass opacification resolved spontaneously, while the underlying focal fibrotic changes persisted. Chemotherapy with cyclophosphamide and doxorubicin may have increased the susceptibility towards radiation-induced lung injury. ${ }^{7}$ The initially observed ground glass changes could have represented subacute radiation-induced lung injury. ${ }^{8}$ However, the temporal relationship between the breath-hold dive and the occurrence of symptoms that did not recur with cessation of diving and swimming practice, as well as the resolution of ground glass opacification over the next months, implicate cold water immersion and breath-hold diving as the major inciting factors in this clinical scenario, rather than subacute radiation-induced lung injury alone.

Pulmonary hemorrhage resulting from decreased elasticity and resistance of the respiratory membrane has also been described in conditions such as Goodpasture's syndrome. ${ }^{9}$ In sum, this case highlights the risk of pulmonary edema in breath-hold diving and swimming and suggests that special caution is warranted among divers who have a history of radiation therapy involving the thorax.

\section{Conclusion}

Breath-hold diving is associated with increased risk of pulmonary edema and hemorrhage in healthy divers due to capillary stress failure. Several mechanisms contribute to redistribution of blood to the pulmonary vasculature and "stress failure" of the pulmonary capillaries due to cold ambient temperature and exertion. Given sufficient diving depth, pulmonary barotrauma of descent can also occur. Taken together, these mechanisms result in increased transcapillary pressure gradient and can lead to "stress failure" of the pulmonary capillaries. The transcapillary pressure gradient can be further increased by diaphragmatic movement against a closed glottis, resulting in negative intraalveolar pressure.
Divers with a history of radiation therapy to the thorax should be cautioned about possible increased risk of focal pulmonary hemorrhage resulting from breath-hold diving, immersion or swimming. Pre-existing focal lung disease and a less resilient respiratory membrane may place these patients at a higher risk of diving- or swimming-induced lung injury, pulmonary hemorrhage, and hemoptysis.

\section{References}

1. Boussuges A, Pinet C, Thomas P, Bergmann E, Sainty JM, Vervloet D. Haemoptysis after breath-hold diving. Eur Respir J 1999; 13:697-699.

2. Lindholm P, Lundgren CE. The physiology and pathophysiology of human breath-hold diving. J Appl Physiol 2009; 106:284-292.

3. West JB, Mathieu-Costello O. Stress failure of pulmonary capillaries: role in lung and heart disease. Lancet 1992; 340:762-767.

4. Keatinge WR, McIroy MB, Godfien A. Cardiovascular response to ice-cold showers. J Appl Physiol 1964; 19:1145-1150.

5. Hodges AN, Mayo JR, McKenzie DC. Pulmonary oedema following exercise in humans. Sports Med 2006; 36:501-512.

6. Kiyan E, Aktas S, Toklu AS. Hemoptysis provoked by voluntary diaphragmatic contractions in breath-hold divers. Chest 2001; 120:2098-2100

7. Movsas B, Raffin TA, Epstein AH, Link CJ Jr. Pulmonary radiation injury. Chest 1997; 111:1061-1076.

8. Ikezoe J, Takashima S, Morimoto S, Kadowaki K, Takeuchi N, Yamamoto T, Nakanishi K, Isaza M, Arisawa J, Ikeda H. CT appearance of acute radiation-induced injury in the lung. AJR Am J Roentgenol 1988; 150:765-770.

9. Donald KJ, Edwards RL, McEvoy JD. Alveolar capillary basement membrane lesions in Goodpasture's syndrome and idiopathic pulmonary hemosiderosis. Am J Med 1975; 59:642-649.

\section{Author Affiliations}

Markus Gutsche, MD*,† and Ware G. Kuschner, MD*,

*Pulmonary Section, US Department of Veterans Affairs Palo Alto Health Care System, Palo Alto, California, USA

tDivision of Pulmonary and Critical Care Medicine, Stanford University School of Medicine, Stanford, California, USA 